\title{
Strongly bound states of hydrogen in intense magnetic field*
}

\author{
J. E. Avron \\ Department of Physics, Technion, Haifa, Israel \\ I. W. Herbst \\ Department of Mathematics, University of Virginia, Charlottesville, Virginia 22903 \\ B. Simon \\ Departments of Mathematics and Physics, Princeton University, Princeton, New Jersey 08540
}

(Received 26 March 1979)

\begin{abstract}
The authors derive asymptotic formulas for the energies of the strongly bound states of hydrogen for large magnetic fields. Rigorous lower bounds on the binding and also upper bounds are given based on generous estimates of the errors. Comparison with variational and other previous numerical results shows their tendency to underestimate the binding.
\end{abstract}

\section{INTRODUCTION}

Intense (sometimes called "superstrong") magnetic fields are characterized by Landau orbits which are of the same order as, or smaller than, the Bohr radius. This is the case for $B \sim 3 \times 10^{9} \mathrm{G}$. Such fields are believed to pervade the surfaces of certain neutron and magnetic stars. ${ }^{1}$ They make spectroscopic and other properties of matter very different from the ordinary. For the obvious reasons of abundancy and theoretical tractability the spectral properties of hydrogen are of particular interest. There is also independent theoretical interest in the problem in the context of perturbation theory..$^{2-4}$

The key to the large-field behavior is the (essentially) one-dimensional dynamics in the direction of $B$. (Schiff and Snyder realized this as early as $1939 . .^{5}$ ) Hence hydrogen in a large magnetic field is related to some form of the one-dimensional Coulomb (long-range) problem. A definite connection with the one-dimensional truncated Coulomb Hamiltonian $[V(x)=-1 /(|x|+a)]$ was suggested by Ruderman in 1971.6 This gave the leading term in the $B \rightarrow \infty$ expansion for the binding energy. However, the connection proposed by Ruderman was just a clever guess. It was not clear how seriously it should be taken, i.e., to what order it is accurate. (This question is answered by the present work.)

Much effort has been devoted to numerical analysis of the problem. Among the methods employed were variational, ${ }^{7,8}$ Padé approximations, ${ }^{9}$ numerical integration of differential equations, ${ }^{10,11}$ and Thomas-Fermi. ${ }^{21}$ Typically the various methods led to scattering of $\sim 20 \%$ in their estimates.

Theoretically the problem was in two parts: first, to perform the Schiff-Snyder reduction to one dimension systematically; second, to find a gener- al method for analyzing the one-dimensional Hamiltonian in the appropriate limit. It turns out that both problems are intimately related. Both can be solved by a theory of weak coupling recently developed by one of us.,4

Before explaining what weak coupling has to do with large magnetic fields let us explain what the strongly bound states are. The strongly bound states are those that increase their binding indefinitely with $B$. On each subspace, with fixed azimuthal quantum number $m$, the ground state is strongly bound. Heuristically this comes about by squashing the state onto the attractive Coulomb singularity. Ruderman found that this divergence of the binding is essentially logarithmic in $B$.

How is weak coupling related to high $B$ ? This is a consequence of the scaling properties of the Hamiltonian $^{17}$ : Surmelian and $\mathrm{O}^{\prime}$ Connell ${ }^{13}$ noted that with charge $z$ the hydrogenlike Hamiltonian $H(B, z)$ satisfies

$$
H(B, z) \cong B H\left(1, z B^{-1 / 2}\right)
$$

$(\cong$ denotes unitary equivalence). Hereafter we use $\lambda$ for $z$. It follows that $B \rightarrow \infty$ corresponds to $\lambda \rightarrow 0$. This is the weak coupling.

The paper is organized as follows. In Sec. II we derive weak coupling formulas and show that the one-dimensional Schiff-Snyder Hamiltonian is a first-order approximation in the weak coupling expansion. In Sec. III we evaluate explicitly the first few terms in the expansion for the Schiff-Snyder Hamiltonian. This yields asymptotic formulas for the binding energies for the ground states for arbitrary quantum numbers $m$. In Sec. IV we bound the first correction due to the latent two dimensions. Section $\mathrm{V}$ is numerical. We give generous upper and rigorous lower bounds for the binding energies. Comparison with other results is also made.

(c) 1979 The American Physical Society 
In Secs. II-IV, the unit of energy is $54.4 \mathrm{eV}$ and the unit of magnetic field $9.4 \times 10^{9} \mathrm{G}$.

\section{WEAK COUPLING}

The Schrödinger equation for (spinless) hydrogen in a constant magnetic field $\vec{B}$ (and infinitely heavy nucleus) is

$$
H(B)=\left(\overrightarrow{\mathrm{p}}-\frac{1}{2} \overrightarrow{\mathrm{B}} \times \overrightarrow{\mathrm{r}}\right)^{2}-1 /|\overrightarrow{\mathrm{r}}| \text {. }
$$

By (1.1), $H(B)$ is essentially equivalent to

$$
\tilde{H}(\lambda)=(\overrightarrow{\mathrm{p}}-\hat{z} \times \overrightarrow{\mathbf{r}})^{2}-\lambda /|\overrightarrow{\mathbf{r}}|,
$$

with $\hat{z}$ a unit vector and

$$
\lambda=(2 / B)^{1 / 2} \text {. }
$$

In each subspace, with azimuthal quantum number $m$, the continuous spectrum of (2.1) starts at $|B|(|m|-m+1) .^{14}$ The binding energy is the (positive) distance of the eigenvalue from the threshold of the continuous spectrum. An index $m$, which we shall drop as a rule, denotes the $L_{\boldsymbol{Z}}=m$ subspace. It is sufficient to consider $m \geqslant 0$, which we henceforth do. Let $\alpha^{2}$ denote the binding energy of (2.2). The corresponding binding for (2.1) will be denoted by $\epsilon^{2}$. The two are related by

$$
\epsilon^{2}=\frac{1}{2} B \alpha^{2} \text {. }
$$

Let

$$
H_{2, m}=(\overrightarrow{\mathrm{p}}-\hat{z} \times \overrightarrow{\mathrm{r}})_{m}^{2}-2 .
$$

The eigenvalue problem $(2.2)$ can be written

$$
[1-\lambda K(\alpha)] \varphi=0,
$$

where

$$
\begin{aligned}
& K(\alpha)=\left(1 /|\overrightarrow{\mathbf{r}}|^{1 / 2}\right)\left(H_{2}+\alpha^{2}\right)^{-1}\left(1 /|\overrightarrow{\mathbf{r}}|^{1 / 2}\right), \\
& \varphi=|\overrightarrow{\mathbf{r}}|^{-1 / 2} \psi ;
\end{aligned}
$$

$\psi$ is the usual bound-state wave function. Now

$$
\left(H_{2}+\alpha^{2}\right)^{-1}=|0\rangle\langle 0| \frac{e^{-\alpha_{1}\left|z-z^{\prime}\right|}}{2 \alpha}+\frac{1-|0\rangle\langle 0|}{H_{2}+\alpha^{2}},
$$

where $|0\rangle$ is the ground state of the two-dimensional Landau Hamiltonian restricted to the $L_{z}=m$ subspace. The first term on the right-hand side of (2.7) is singular in the limit $\alpha \rightarrow 0$. The second term, on the other hand, is uniformly bounded by $\frac{1}{4}$. Motivated by this and Ref. 4 , we introduce a more elaborate decomposition. Let

$$
\begin{aligned}
L= & \frac{1}{|\overrightarrow{\mathbf{r}}|^{1 / 2}} \frac{1-|0\rangle\langle 0|}{H_{2}+\alpha^{2}} \frac{1}{|\overrightarrow{\mathbf{r}}|^{1 / 2}}, \\
P= & \frac{1}{2} \frac{1}{|\overrightarrow{\mathrm{r}}|^{1 / 2}}|0\rangle\langle 0| e^{-\alpha|z|-\alpha\left|z^{\prime}\right|} \frac{1}{|\overrightarrow{\mathbf{r}}|^{1 / 2}}, \\
Q= & \frac{1}{2} \frac{1}{|\overrightarrow{\mathbf{r}}|^{1 / 2}}|0\rangle\langle 0|\left(e^{-\alpha\left|z-z^{\prime}\right|}-e^{-\alpha\left|z+z^{\prime}\right|}\right) \\
& \times \frac{\theta\left(z z^{\prime}\right)}{|\overrightarrow{\mathbf{r}}|^{1 / 2}}
\end{aligned}
$$

and $\theta(x)=1$ for $x \geqslant 0$ and zero otherwise. Then

$$
K=P / \alpha+Q / \alpha+L \text {. }
$$

By Appendix A

$$
\|L\| \leqslant 3 \pi / 4 \sqrt{2} \text {. }
$$

Thus for $B \geqslant 6[B$ and $\lambda$ related by (2.3)] $\lambda\|L\|<1$. By direct variation calculation (see Appendix A) one can show that the ground state in the $m$ subspace has binding that increases without limit as $B \rightarrow \infty$. In addition, it is shown that

$$
\|Q\| \leqslant \sqrt{3} \text {. }
$$

Consequently $(\lambda / \alpha) Q=(1 / \epsilon) Q$ is arbitrarily small for sufficiently large $B$. Thus, as in the one-dimensional case, ${ }^{3,4}$ the key to the analysis is the realization that the most divergent part of $K$ as $\alpha$ $\rightarrow 0$ is rank 1 .

Let $B_{c}$ be so large ( $\lambda$ so small) that

$$
\|\lambda L+(1 / \epsilon) Q\|<1
$$

for $B \geqslant B_{c}$. By Appendix A and the numerical results of Sec. IV, one finds $B_{c} \leqslant 300$.

Theorem 2.1: The eigenvalue equation for the ground state with binding $\epsilon^{2}$ for $B \geqslant B_{c}$ is given by

$$
\epsilon=\operatorname{Tr}\left\{[1-(1 / \epsilon) Q-\lambda L]^{-1} P\right\} .
$$

Proof: Since $[1-(1 / \epsilon) Q-(1 / \lambda) L] \dot{\psi}=0$ implies $\psi \equiv 0$ for $B \geqslant B_{c},(2.5)$ can be written

$$
[1-(1 / \epsilon) A] \varphi=0,
$$

where

$$
A \equiv[1-(1 / \epsilon) Q-\lambda L]^{-1} P .
$$

$A$ is a product of a bounded operator and a rank-1 operator. By standard arguments the eigenvalue problem (2.14) has the eigenvalue equation

$$
\operatorname{det}[1-(1 / \epsilon) A]=1-(1 / \epsilon) \operatorname{Tr}(A)=0,
$$

since $A$ is rank 1, Q.E.D.

A systematic expansion in the small parameters $\lambda$ and $1 / \epsilon$ follows from (2.15) by expanding the denominator in a power series to lower orders:

$\epsilon=\operatorname{Tr}(P)+(1 / \epsilon) \operatorname{Tr}(P Q)+(2 / B)^{1 / 2} \operatorname{Tr}(L P) \cdots$.

In Sec. III we evaluate the first two traces (the leading behavior) and in Sec. IV we bound the third.

Setting $L=0$ in (2.16) gives the eigenvalue equation for the ground state of the Schiff-Snyder Hamiltonian.

\section{SCHIFF-SNYDER HAMILTONIAN}

The Schiff-Snyder Hamiltonian $\tilde{H}_{\mathrm{S}}$ is

$$
\tilde{H}_{\mathrm{S}}^{(m)}=-\frac{d^{2}}{d x^{2}}-\lambda W_{m}(x),
$$


where

$$
W_{m}(x) \equiv\left\langle 0\left|\frac{1}{|\overrightarrow{\mathrm{r}}|}\right| 0\right\rangle=\frac{1}{|m| !} \int_{0}^{\infty} \frac{e^{-t} t^{|m|} d t}{\left(t+x^{2}\right)^{1 / 2}} .
$$

Proposition 3.1: The eigenvalues of $\tilde{H}_{\mathrm{S}}^{(m)}(\lambda)$ are bounded from above by the eigenvalues of the onedimensional truncated Coulomb Hamiltonian with potential

$$
-\lambda /\left[|x|+(m+1)^{1 / 2}\right]
$$

and

$$
E_{n}^{\text {Hyd }} \leqslant E_{2 n}^{\text {Schiff-Snyd }},
$$

with $E_{n}^{\text {Hyd }}$ the $n$th $L_{z}=0$ hydrogenic energy (with $\lambda$ $=e^{2}$ ).

Proof:

$$
1 /\left(m+1+x^{2}\right)^{1 / 2} \leqslant W_{m}(x) \leqslant 1 /|x| .
$$

The right-hand side of (3.3) follows directly from (3.2) and the left-hand side, which is also easy, is proved in Appendix C. It follows that $W_{m}(x)$ $\geqslant\left[(m+1)^{1 / 2}+|x|\right]^{-1}$. This together with the minmax principle completes the proof of the first half of the proposition. For the second half, note that the one-dimensional Schrödinger equation with Dirichlet boundary conditions is identical to the radial wave equation with $l=0$. Q.E.D.

Corollary 3.2: Only the ground state of $\tilde{H}_{\mathrm{S}}$ is strongly bound in the sense that $\alpha / \lambda$ goes to infinity as $\lambda \rightarrow 0$.

Let $\alpha^{2}$ be the binding energy for the ground state of $\tilde{H}_{\mathrm{S}}$. Mimicking the derivation in Sec. II, we get

$$
\alpha=\lambda \operatorname{Tr}(P)+\frac{\lambda^{2}}{\alpha} \operatorname{Tr}(P Q)+\frac{\lambda^{3}}{\alpha^{2}} \operatorname{Tr}\left(P Q^{2}\right)+\cdots,
$$

where $P$ and $Q$ are as in (2.8). This is identical to (2.16), with $\lambda$ and $\alpha$ related to $B$ and $\epsilon$ by (2.3) and (2.4) and $L$ set equal to zero. Now

$$
\begin{aligned}
\operatorname{Tr}(P)_{m}= & \int_{0}^{\infty} e^{-2 \alpha x} W_{m}(x) d x \\
= & \int_{2 \alpha}^{\infty} \frac{e^{-x}}{x} d x+\int_{0}^{1}(1-2 \alpha x) W_{m}(x) d x \\
& +\int_{1}^{\infty} e^{-2 \alpha x}\left(W_{m}-\frac{1}{x}\right) d x+O(\alpha) .
\end{aligned}
$$

Using the identities ${ }^{24}[(3.7)$ follows from (3.8)]

$$
\begin{aligned}
\int_{1}^{\infty} & \left(W_{m}(x)-\frac{1}{x}\right) d x \\
& =\ln 2-\frac{1}{m !} \int_{0}^{\infty} e^{-x} x^{m} \ln \left[1+(1+x)^{1 / 2}\right] d x, \\
\int_{0}^{1} & W_{m}(x) d x \\
& =\frac{1}{m !} \int_{0}^{\infty} e^{-x} x^{m} \ln \left[1+(1+x)^{1 / 2}\right] d x+\frac{1}{2}\left(\gamma-q_{m}\right),
\end{aligned}
$$

$$
\begin{aligned}
& \int_{0}^{\infty} e^{-x} x^{m} \ln \frac{1}{x} d x=m !\left(\gamma-q_{m}\right), \\
& q_{m} \equiv \sum_{i=1}^{m} \frac{1}{i}, \quad q_{0}=0, \\
& \int_{0}^{\infty}\left[x W_{m}(x)-1\right] d x=-\left(m+\frac{1}{2}\right) / m !, \\
& \int_{2 \alpha}^{\infty} \frac{e^{-x}}{x} d x=-\gamma-\ln (2 \alpha)-\sum_{n=1}^{\infty}(-1)^{n} \frac{(2 \alpha)^{n}}{n(n !)},
\end{aligned}
$$

with $\gamma$ being Euler's constant, one gets

$\operatorname{Tr}(P)=\ln (1 / \alpha)-\frac{1}{2}\left(\gamma+q_{m}\right)+2 \alpha\left(m+\frac{1}{2}\right) / m !+O(\alpha)$.

For the next term

$$
\begin{aligned}
\operatorname{Tr}(P Q)_{m}= & \frac{1}{2} \int_{0}^{\infty} d x d y W_{m}(x) e^{-\alpha x} W_{m}(y) e^{-\alpha y} \\
& \times\left(e^{-\alpha|x-y|}-e^{-\alpha|x+y|}\right) \\
= & \int_{0}^{\infty} W_{m}(x) e^{-2 \alpha x} d x \int_{0}^{x} d y W(y) \\
& -\frac{1}{2}\left(\int_{0}^{\infty} e^{-2 \alpha x} W_{m}(x) d x\right)^{2} \\
& =\int_{0}^{\infty} W_{m}(x) e^{-2 \alpha x} \int_{0}^{x} W_{m}(y)\left(1-e^{-2 \alpha y}\right) d y
\end{aligned}
$$

It is easy to show (see below) that

$$
0 \leqslant \operatorname{Tr}(P Q) \leqslant \frac{1}{12} \pi^{2} \text {. }
$$

Let

$$
\begin{aligned}
& \varphi(x)=-\frac{1}{m !} \int_{0}^{\infty} \frac{e^{-t} t^{m} d t}{\left(4 \alpha^{2} t+x^{2}\right)^{1 / 2}}, \\
& \xi(x)=\int_{0}^{x}\left(1-e^{-y}\right) \varphi(y) d y, \\
& \chi(x)=\int_{0}^{x} \frac{1-e^{-y}}{y} d y, \\
& \eta(x)=\xi(x)-\chi(x) .
\end{aligned}
$$

Then

$$
\eta(x)=\int_{0}^{x}[t \varphi(t)-1] d t+O(\alpha)
$$

and

$$
\begin{aligned}
\operatorname{Tr}(P Q)= & \int_{0}^{\infty} \frac{e^{-x} \chi(x)}{x} d x+\int_{1}^{\infty} \frac{e^{-x}}{x} \eta(x) \\
& +\int_{0}^{1} e^{-x}\left(\varphi-\frac{1}{x}\right) \chi(x) d x+\int_{0}^{1} \frac{e^{-x}}{x} \eta(x) d x \\
& +\int_{0}^{1} \frac{e^{-x}}{x}\left(\varphi-\frac{1}{x}\right) \eta(x) d x+O(\alpha)
\end{aligned}
$$


These integrals are evaluated in Appendix B. The result is

$$
\begin{aligned}
\operatorname{Tr}(P Q)=\frac{\pi^{2}}{12} & +\alpha \frac{\left(m+\frac{1}{2}\right) !}{m !} \ln \left(\alpha^{2}\right) \\
& +\frac{\left(m+\frac{1}{2}\right) !}{m !} D_{m} \alpha+\alpha f_{m},
\end{aligned}
$$

where

$$
\begin{aligned}
& D_{m}=q_{m+1 / 2}+2 \ln (2)-2+\gamma-2 c_{1}, \\
& c_{1}=\int_{0}^{\infty}\left[y\left(y^{2}+1\right)^{-1 / 2}-1\right] \ln y d y \simeq 0.30685 ;
\end{aligned}
$$

$q_{m}$ is as in (3.8) (see Table I), $f_{m}$ is the constant

$$
f_{m}=\int_{0}^{\infty}\left(W_{m}(x)-\frac{1}{x}\right) d x \int_{0}^{x} d y\left[W_{m}(y)-1\right] .
$$

TABLE I. Constants $D_{m}$ and $q_{m}$ of Eqs. (3.18) and (3.19) computed for angular momenta $m, m=0,1,2$.

\begin{tabular}{ccc}
\hline \hline$m$ & $q_{m+1 / 2}$ & $D_{m}$ \\
\hline 0 & $2(1-\ln 2)$ & $\gamma-2 C_{1}=-0.03648$ \\
1 & $2\left(\frac{4}{3}-\ln 2\right)$ & $\frac{2}{3}+\gamma-2 C_{1}=0.6302$ \\
2 & $2\left(\frac{23}{15}-\ln 2\right)$ & $\frac{16}{15}+\gamma-2 C_{1}=1.0302$ \\
\hline \hline
\end{tabular}

In Appendix $\mathrm{C}$ we prove

$$
0 \leqslant f_{m} \leqslant 2(m+1)^{1 / 2}(1-\ln 2) .
$$

We have made no attempt to evaluate $f_{m}$ numerically. For the third-order term we have the upper bound

$$
\begin{aligned}
\operatorname{Tr}\left(P Q^{2}\right)= & \iint_{\substack{x_{3} \geq 0 \\
x_{1} \geq x_{2} \geq 0}} W\left(x_{1}\right) W\left(x_{2}\right) W\left(x_{3}\right) e^{-\alpha x_{1}-\alpha x_{2}}\left(e^{-\alpha\left|x_{2}-x_{3}\right|}-e^{-\alpha\left|x_{2}+x_{3}\right|}\right)\left(e^{-\alpha\left|x_{3}-x_{1}\right|}-e^{-\alpha\left|x_{3}+x_{1}\right|}\right) \\
= & \iint_{x_{1} \geq x_{2} \geq x_{3} \geq 0} \int_{\substack{1\\
}} d x_{1} d x_{2} d x_{3} W\left(x_{1}\right) W\left(x_{2}\right) W\left(x_{3}\right) \\
& \quad \times\left[\exp \left(-2 \alpha x_{1}-2 \alpha x_{2}\right) \sinh ^{2}\left(\alpha x_{3}\right)+2 \exp \left(-2 \alpha x_{1}-\alpha x_{2}-\alpha x_{3}\right) \sinh \left(\alpha x_{3}\right) \sinh \left(\alpha x_{2}\right)\right] .
\end{aligned}
$$

By (3.3)

$$
0 \leqslant \operatorname{Tr}\left(P Q^{2}\right) \leqslant \iint_{x_{1} \geq x_{2} \geq x_{3}}\left(x_{1} x_{2} x_{3}\right)^{-1}\left[e^{-2 x_{1}-2 x_{2}} \sinh ^{2}\left(x_{3}\right)+2 e^{-2 x_{1}-x_{2}-x_{3}} \sinh \left(x_{2}\right) \sinh \left(x_{3}\right)\right] \leqslant \frac{5}{16}
$$

[use $e^{-x} \sinh (x) \leqslant x$ ].

Collecting these terms, we obtain an asymptotic implicit function for the ground-state eigenvalue for the Schiff-Snyder Hamiltonian:

$$
\begin{aligned}
\frac{\alpha_{m}}{\lambda}= & \ln \frac{1}{\alpha_{m}}-\frac{1}{2}\left(\gamma+q_{m}\right)+\frac{\pi^{2} \lambda}{12 \alpha}+\lambda \ln \left(\alpha_{m}^{2}\right) \\
& +\frac{2 \alpha\left(m+\frac{1}{2}\right) !}{m !}+\frac{\lambda D_{m}\left(m+\frac{1}{2}\right) !}{m !}+\text { error } .
\end{aligned}
$$

The error is roughly of the order of $\frac{5}{16}(\lambda / \alpha)^{2}$.

Strictly speaking, only the first three terms on the right-hand side of $(3.25)$ are significant. $\alpha / \lambda$ is of the order of $\ln (1 / \lambda)$, and so the error dominates the $\lambda \ln \left(\alpha^{2}\right)$ term and the two following terms for $\lambda$ sufficiently small. The main reason why we retain these terms is that we have been able to compute them. We mention, however, that these are also the first terms that distinguish the SchiffSnyder energy from the truncated Coulomb. Another reason for retaining the high-order terms is that, in the range of interest, $\lambda$ is not so small that these terms are dominated by the error term.

\section{ASYMPTOTIC FORMULA}

A. Latent two dimensions

By (2.17) the lowest-order correction taking into account the two dimensions transverse to $B$ is $(2 / B)^{1 / 2} \operatorname{Tr}(L P)$.

$$
\begin{aligned}
\operatorname{Tr}(L P)= & \sum_{n=1}^{\infty} \int \frac{W_{n .0}(x) W_{0 . n}\left(x^{\prime}\right)}{2\left(\alpha^{2}+4 n\right)^{1 / 2}} \\
& \times \exp \left[-\left(\alpha^{2}+4 n\right)^{1 / 2}\left|x-x^{\prime}\right|\right] \\
= & \sum_{n=1}^{\infty}\left(\frac{\pi}{2}\right)^{1 / 2} \int \frac{\left|\hat{W}_{n .0}(p)\right|^{2} d p}{p^{2}+4 n+\alpha^{2}},
\end{aligned}
$$

where

$$
W_{n, 0}(z)=e^{-\alpha|z|}\left\langle n\left|\frac{1}{|\overrightarrow{\mathrm{r}}|}\right| 0\right\rangle
$$

and a caret denotes Fourier transform. Hence 


$$
\begin{aligned}
\operatorname{Tr}(L P) & \leqslant \sum_{1}^{\infty}\left(\frac{\pi}{2}\right)^{1 / 2} \int \frac{\left|\hat{W}_{n, 0}(p)\right|^{2}}{p^{2}+4} d p \leqslant\left(\frac{\pi}{2}\right)^{1 / 2} \frac{1}{4} \sum_{1}^{\infty} \int\left|W_{n, 0}(x)\right|^{2} d x \\
& =\frac{1}{4}\left(\frac{\pi}{2}\right)^{1 / 2} \int_{-\infty}^{\infty} d x e^{-2|x|} \int_{0}^{\infty} d t \frac{e^{-t} t^{m}}{m !\left(t+x^{2}\right)} \leqslant \frac{1}{2}\left(\frac{\pi}{2}\right)^{3 / 2} \frac{\left(m-\frac{1}{2}\right) !}{m !} \leqslant \pi .
\end{aligned}
$$

\section{B. Main result}

From (3.25), for the Schiff-Snyder one-dimensional Hamiltonian, (2.3), (2.4), and (4.3) we obtain the ground state of the Hamiltonian (2.1), which, for $B \geqslant B_{c}$, is given by the asymptotic formula

$$
\begin{aligned}
\epsilon_{m}= & \frac{1}{2} \ln \left(\frac{B}{\epsilon_{m}^{2}}\right)-\frac{1}{2}\left(\gamma+q_{m}+\ln 2\right)+\frac{\pi^{2}}{12 \epsilon_{m}} \\
& -\left(\frac{2}{B}\right)^{1 / 2} \ln \left(\frac{B}{2 \epsilon_{m}^{2}}\right)+2\left(\frac{2}{B}\right)^{1 / 2} \epsilon_{m} \frac{\left(m+\frac{1}{2}\right) !}{m !} \\
& +\left(\frac{2}{B}\right)^{1 / 2} \frac{\left(m+\frac{1}{2}\right) ! D_{m}}{m !}+\text { error }
\end{aligned}
$$

[ $q_{m}$ and $D_{m}$ are given in (3.8) and (3.19)]. The error is roughly

$$
\text { Error } \sim \frac{5}{4} \ln ^{-2} B+\left(2 \pi^{2} / B\right)^{1 / 2}
$$

The first term is the "one-dimensional error" (3.24) and the second the "two-dimensional" (4.3). For magnetic fields $B \lessgtr 3000$, (4.3) dominates (3.24). (The converse holds for higher $B$.) It follows that by computing a few terms in (4.1) the numerical accuracy of (4.4) would improve substantially.

\section{NUMERICAL RESULTS}

The physically interesting range of intense magnetic fields is restricted to fields smaller than a few thousands in the $9.4 \times 10^{9}-\mathrm{G}$ unit. In this range the relevant small parameters are not very small. Consequently the weak coupling expansion to the order given in Eq. (4.4) is only moderately accurate and the error terms, $B^{-1 / 2}$ and $\ln ^{-2} B$ [see Eq. (4.5)], are not negligible. We shall describe the solutions of Eq. (4.4), discuss their accuracy, and compare the results to those of other works.

We denote the binding energy obtained by solving the implicit function $E_{m}(4.4)$. We also consider shorter series of the weak coupling expansion. In particular, $E_{m}(2)$ denotes the binding energy for the weak coupling formula in which only the first two leading terms in the right-hand side of Eq. (4.4) are kept. The behavior of the sequence of numbers obtained as more terms in the series in Eq. (4.4) are retained is an empirical measure of the accuracy of $E_{m}$. Since $E_{m}(3)$ turns out to be quite close to $E_{m}$, it seems that the distance between $E_{m}(2)$ and $E_{m}$ should be an empirically safe estimate of the accuracy of $E_{m} . E_{m}$ and $E_{m}(2)$ are given in Table II for $m=0,1$ in units of $13.6 \mathrm{eV}$. [ $E_{m}$ turns to be insensitive to $f_{m}$ within the range given in (3.22).] The table indicates only a $20 \%$ accuracy for $m=0$ and $B \sim 1000$. In Table I we have also given a rigorous lower bound on the binding which follows from the truncated Coulomb (Prop. 3.1). This bound is not expected to be optimal. The eigenvalues of the truncated Coulomb Hamiltonian are given by the zeros of a certain hypergeometric function ${ }^{15,16}$ (see Appendix E). These have been determined numerically to an accuracy better than 0.001 .

Table III considers the $m=0$ ground state. The table contains results obtained by alternative methods in other works (Refs. 7, 8, 9, 11, 12, 19, 20, 22): a rigorous lower bound on the binding, and a generous upper bound. This table is also in units of 13.6 $\mathrm{eV}$ and $2.35 \times 10^{9} \mathrm{G}$ (unlike the other sections of this work). The lower bound has been discussed above. The upper bound was obtained by taking the generous bounds (3.24), (4.3), and (D3) for $\operatorname{Tr}\left(P Q^{2}\right), \operatorname{Tr}(L P)$, and $\operatorname{Tr}(P Q)$ in (2.16).

TABLE II. Binding energy in units of $13.6 \mathrm{eV}$ for the tightly bound states with $m=0$ and $m=1$ in magnetic fields in the $9.4 \times 10^{9}-\mathrm{G}$ unit. $E_{0}(2)$ and $E_{1}(2)$ are the solutions of the weak coupling equation to the leading two orders. $E_{0}$ and $E_{2}$ are the solutions of the full weak coupling equations (4.4) for $m=0$ and $m=1$.

\begin{tabular}{rrrrrrr}
\hline \hline & \multicolumn{3}{c}{$\begin{array}{l}\text { Lower } \\
\text { bound }\end{array}$} & $E_{0}(2)$ & \multicolumn{3}{c}{$E_{0}$} & $\begin{array}{r}\text { Lower } \\
\text { bound }\end{array}$ & $E_{1}(2)$ & \multicolumn{1}{c}{$E_{1}$} \\
\hline 25 & 4.31 & 4.34 & 7.78 & 3.38 & 2.97 & 8.96 \\
50 & 5.44 & 5.44 & 9.06 & 4.30 & 4.13 & 8.50 \\
100 & 6.83 & 7.40 & 11.00 & 5.46 & 4.94 & 9.45 \\
300 & 9.63 & 11.44 & 15.21 & 7.77 & 7.71 & 12.32 \\
500 & 11.23 & 13.76 & 17.64 & 9.11 & 9.49 & 14.12 \\
851 & 13.10 & 16.48 & 20.49 & 10.70 & 11.62 & 16.30 \\
1000 & 13.72 & 17.38 & 21.42 & 11.22 & 12.33 & 17.02 \\
2000 & 16.65 & 21.58 & 25.79 & 13.72 & 15.70 & 20.47 \\
3000 & 18.56 & 24.32 & 28.62 & 15.38 & 17.94 & 22.75 \\
\hline \hline
\end{tabular}


TABLE III. Binding energy for the $m=0$ ground state in units of $13.6 \mathrm{eV}$ and magnetic fields in the $2.35 \times 10^{9}-\mathrm{G}$ unit. The "Lower bound" column is the solution of the truncated Coulomb Hamiltonian; CK-data of Canuto and Kelly (Ref. 11); CLR-Cohen, Lodenquai, and Ruderman (Ref. 19); RS-Rau, Spruch, and Mueller (Refs. 8,12); SHSO-Smith, Henry, Surmelian, and O'Connell (Ref. 7, 22); AHS-the present work.

\begin{tabular}{|c|c|c|c|c|c|c|c|c|c|}
\hline$B$ & $\begin{array}{l}\text { Lower } \\
\text { bound }\end{array}$ & $\mathrm{CK}$ & CLR & $L$ & $P G$ & $\mathrm{RS}$ & SHSO & AHS & $\begin{array}{l}\text { Upper } \\
\text { bound }\end{array}$ \\
\hline 25 & 2.63 & & & 4.73 & 5.33 & & & & 13 \\
\hline 50 & 3.38 & & & & & & & 7.77 & 14 \\
\hline 100 & 4.31 & & 6.47 & 7.46 & 8.54 & 6.93 & 7.56 & 7.78 & 15 \\
\hline 300 & 6.23 & & $10.15^{\mathrm{a}}$ & & & 9.81 & 10.57 & 10.13 & 18 \\
\hline 500 & 7.34 & & & & & & & 11.75 & 20 \\
\hline 852 & 8.67 & 14 & $13.6^{\mathrm{a}}$ & & & & & 13.75 & 22 \\
\hline 1000 & 9.12 & & $14.4^{\mathrm{a}}$ & & 16.8 & 14.1 & & 14.42 & 23 \\
\hline 2000 & 11.23 & & & & & & & 17.64 & 27 \\
\hline 3000 & 12.64 & & & & & & & 19.78 & 30 \\
\hline
\end{tabular}

${ }^{a}$ See also Ref. 9.

A direct check on the accuracy of the method of weak coupling is presented in Table IV. The table compares $\alpha$, the square root of the binding [in $\left.(54.4 \mathrm{eV})^{1 / 2}\right]$ for the truncated Coulomb obtained by weak coupling, with the exact results. The small coupling constant $\lambda$ was chosen to correspond [see Eq. (2.3)] to $100 \leqslant B \leqslant 1000$. The weak coupling formula for the truncated Coulomb Hamiltonian is given in Eq. (E5) in Appendix E. In Appendix E the exact solution is also reviewed. Table IV provides an indirect method for estimating the accuracy of Eq. (4.4), $16 \%$ accuracy at $B \sim 100$ and $7 \%$ accuracy at $B \sim 1000$. This error is smaller than the two estimates of the error given above and it is possible that the extra two dimensions for the Zeeman Hamiltonian increase the error.
In conclusion it seems to us that Tables $I I$ and III provide a realistic estimate of $E_{m}$ and its accuracy.

\section{ACKNOWLEDGMENTS}

The work of one of us (I.W.H.) was supported by the NSF under Grant No. MCS-78-00101; one of us (B.S.) was partially supported by NSF Grant No. MCS-78-01885. Publication partially supported by NSF Grant No. PHY-78-23952.

\section{APPENDIX A}

a. $\|L\|$. Since $H_{2}(1-|0\rangle\langle 0|) \geqslant 4$ and $1 / a \leqslant \frac{3}{2}(1 /$ $a+2)$ for $a \geqslant 2$, we have the operator identity

$$
|L| \leqslant \frac{3}{2}\left(\frac{1}{|\overrightarrow{\mathrm{x}}|^{1 / 2}}\right)\left(H_{2}+2\right)^{-1} \frac{1}{|\overrightarrow{\mathrm{x}}|^{1 / 2}} .
$$

Thus

$$
\|L\| \leqslant \frac{3}{2}\left\||\overrightarrow{\mathrm{x}}|^{-1 / 2}\left(H_{2}+2\right)^{-1}\right\|^{2} \leqslant \frac{3}{2}\left\||\overrightarrow{\mathrm{x}}|^{-1 / 2}|\overrightarrow{\mathrm{p}}|^{-1 / 2}\right\|\left\|\left(p^{2}\right)^{1 / 4}\left(H_{2}+2\right)^{-1 / 4}\right\|^{2}\left\|\left(H_{2}+2\right)^{-1 / 4}\right\|^{2} \leqslant \frac{3}{2} \pi / 2^{3 / 2}
$$

The norm of the first term is $\left(\frac{1}{2} \pi\right)^{1 / 2}$, by Ref. 18 . The others are elementary.

b. $\|Q\|$. Consider a unitarity-dilated $Q$ in the $z$ direction. As $\alpha \rightarrow 0$ it converges to (and is pointwise bounded by)

$$
\begin{aligned}
\tilde{Q}_{0}= & \frac{1}{2}|z|^{-1 / 2}\left(e^{-\left|z-z^{\prime}\right|}-e^{-\left|z+z^{\prime}\right|}\right)\left|z^{\prime}\right|^{-1 / 2} \\
& \times \theta\left(z z^{\prime}\right)|0\rangle\langle 0| .
\end{aligned}
$$

Let

$$
\begin{aligned}
& V_{R}(z) \equiv\left\{\begin{array}{l}
|z|^{-1 / 2}, \quad|z| \leqslant R, \\
0, \quad|z|>R,
\end{array}\right. \\
& V_{\infty}(z) \equiv|z|^{-1 / 2}-V_{R}(z), \\
& A \equiv \frac{1}{2}\left(e^{-\left|z^{-} z^{\prime}\right|}-e^{-\left|z+z^{\prime}\right|}\right) \theta\left(z z^{\prime}\right) .
\end{aligned}
$$

Then

$$
\left\|\tilde{Q}_{0}\right\| \leqslant\left\|V_{R} A V_{R}\right\|+2\left\|V_{R} A V_{\infty}\right\|+\left\|V_{\infty} A V_{\infty}\right\| ;
$$

now

$$
\begin{gathered}
\left\|V_{\infty} A V_{\infty}\right\| \leqslant 1 / R, \\
\left\|V_{\infty} A V_{R}\right\|^{2} \leqslant\left\|V_{\infty} A V_{R}\right\|_{H S}^{2} \\
=\int_{0}^{R} d z \frac{\sinh ^{2}(z)}{z} \int_{R}^{\infty} \frac{e^{-2 x}}{x} d x .
\end{gathered}
$$

Since $\sinh x$ is convex

$$
e^{z}-e^{-z} \leqslant(z / R) e^{R}, \quad 0 \leqslant z \leqslant R .
$$

This gives

$$
\left\|V_{\infty} A V_{R}\right\| \leqslant 1 / 4 \sqrt{R},
$$


TABLE IV. Square root of the binding energy $\alpha$ in units of $(54.4 \mathrm{eV})^{1 / 2}$ for small coupling constants $\lambda$ for the truncated Coulomb.

\begin{tabular}{|c|c|c|}
\hline$\lambda$ & $\begin{array}{c}\alpha \\
\text { Weak } \\
\text { coupling }\end{array}$ & $\begin{array}{c}\alpha \\
\text { Exact }\end{array}$ \\
\hline 0.1414 & 0.171 & 0.1848 \\
\hline 0.1000 & 0.137 & 0.1458 \\
\hline 0.0840 & 0.122 & 0.1293 \\
\hline 0.0632 & 0.101 & 0.1060 \\
\hline 0.0447 & 0.080 & 0.0828 \\
\hline
\end{tabular}

$$
\begin{aligned}
\left\|V_{R} A V_{R}\right\|^{2} & \leqslant\left\|V_{R} A V_{R}\right\|_{H S}^{2} \\
& =2 \int_{0}^{R} d x \frac{e^{-2 x}}{x} \int_{0}^{x} \frac{\sinh ^{2}(y)}{y} d y \\
& \leqslant \frac{1}{2} \int_{0}^{R} e^{-2 x} d x \int_{0}^{x} e^{2 y} d y \leqslant \frac{1}{4} R .
\end{aligned}
$$

So finally

$$
\left\|\tilde{Q}_{0}\right\| \leqslant 3 / 2 \sqrt{R}+\frac{1}{2} \sqrt{R}, \quad R>0 .
$$

(A13) is minimized by $R=3$ :

$$
\left\|\tilde{Q}_{0}\right\| \leqslant \sqrt{3} \text {. }
$$

Lemma A.1: In each subspace with fixed $m$ the ground state of (2.1) has binding energy $\epsilon_{m}^{2}(B)$, which diverges as $B \rightarrow \infty$.

Proof: Let $\beta$ be the unique solution of the implicit function

$$
\beta=2 \lambda(m+1)^{1 / 2} e^{2 \beta} \epsilon_{1}(2 \beta),
$$

with $\epsilon_{1}(x)$ the exponential integral and $\lambda=(2 / B)^{1 / 2}$. As $\lambda \rightarrow 0$,

$$
\beta \sim 2 \lambda(m+1)^{1 / 2} \ln (1 / \lambda) .
$$

Now choose a trial wave function

$$
\exp \left[-\beta|x|(m+1)^{-1 / 2}\right]|0\rangle_{m}
$$

and let $\alpha=\lambda \epsilon$. Using (C3) and the min-max principle, we find

$$
\alpha \geqslant \beta \text {. }
$$

\section{APPENDIX B}

1. $\int_{0}^{\infty}\left[e^{-x} / x\right] \chi(x) d x$

Write $\left(1-e^{-y}\right) / y$ as a power series and integrate term by term. This gives ${ }^{24}$

$$
\begin{gathered}
\int_{0}^{\infty} \frac{e^{-x}}{x} d x \int_{0}^{x} \frac{1-e^{-y}}{y} d y \\
=1-\frac{1}{2^{2}}+\frac{1}{3^{2}}-\frac{1}{4^{2}} \cdots=\frac{\pi^{2}}{12} . \\
\left.2 . \int_{0}^{\infty} / t\left(1+t^{2}\right)^{-1 / 2}-1\right] \ln t d t \\
\int_{0}^{\infty}\left[t\left(1+t^{2}\right)^{-1 / 2}-1\right] \ln t d t \\
=\int_{0}^{1}\left[t\left(1+t^{2}\right)^{-1 / 2}-1\right] \ln t d t \\
+\int_{0}^{1}\left[1-\left(1+t^{2}\right)^{-1 / 2}\right] \frac{\ln t}{t^{2}} d t .
\end{gathered}
$$

Expand the square root by the binomial and integrate term by term. This gives

$$
\begin{aligned}
1- & \frac{1}{2^{2}}-\frac{1}{2}\left(1-\frac{1}{4^{2}}\right)+\frac{1 \times 3}{2 \times 4}\left(\frac{1}{3^{2}}-\frac{1}{6^{2}}\right)-\frac{1 \times 3 \times 5}{2 \times 4 \times 6} \\
& \times\left(\frac{1}{5^{2}}-\frac{1}{8^{2}}\right)+\cdots \\
& \simeq 0.30685 \ldots
\end{aligned}
$$

\section{3. $\int_{0}^{1}\left[e^{-x} / x\right] \eta(x) d x$}

Write $e^{-x} / x$ as an infinite series and integrate by parts. The series obtained is expressible in terms of the exponential integral. It gives

$$
\begin{gathered}
\int_{0}^{1} \frac{e^{-x}}{x} \eta(x) d x=2\left[\gamma+\epsilon_{1}(1)\right] \alpha \frac{\left(m+\frac{1}{2}\right) !}{m !} \\
-\int_{0}^{1} \ln x[x \varphi(x)-1] d x+O(\alpha) .
\end{gathered}
$$

In (B4) a scaled version of (3.9) has been used. Now

$$
\begin{aligned}
\int_{0}^{1} \frac{t \ln t}{\left(a^{2}+t^{2}\right)^{1 / 2}} d t= & a \ln a \int_{0}^{1 / a} \frac{t d t}{\left(t^{2}+1\right)^{1 / 2}}+a \int_{0}^{1 / a} \frac{t \ln t}{\left(t^{2}+1\right)^{1 / 2}} d t+a \int_{0}^{1 / a} \ln t d t \\
& +a \int_{0}^{\infty}\left[t\left(1+t^{2}\right)^{-1 / 2}-1\right] \ln t d t+O(\alpha) \\
= & -a \ln a-1-c_{1}+O(a),
\end{aligned}
$$


where $c_{1}$ is as in Eq. (3.20) above. It follows that

$$
\begin{aligned}
\int_{0}^{1} x \ln x \varphi(x) d x= & -2 \alpha \ln (2 \alpha) \frac{\left(m+\frac{1}{2}\right) !}{m !} \\
& -\frac{\alpha}{m !} \int_{0}^{\infty} e^{-t} t^{m+1 / 2} \ln t d t \\
& -1+2 \alpha c_{1} \frac{\left(m+\frac{1}{2}\right) !}{m !}+O(\alpha) .
\end{aligned}
$$

One can calculate $q_{m+1 / 2}$ exactly using formula 4.333 of Ref. (24) (p. 574):

$$
\begin{gathered}
-\gamma-q_{m-1 / 2}=\left.\frac{\pi^{1 / 2}}{\left(m-\frac{1}{2}\right) !}\left(-\frac{d}{d a}\right)^{m} \frac{\gamma+\ln (4 a)}{a^{1 / 2}}\right|_{a=1} . \\
\text { 4. } \int_{1}^{\infty} e^{-x} / x d x \int_{0}^{x}[y \varphi(y)-1] d y
\end{gathered}
$$

The domain of integration is as in Fig. 1. Since $|y \varphi(y)-1| \leqslant$ const $\alpha^{2} / y^{2}$,

$$
\begin{gathered}
\int_{1}^{\infty} \frac{e^{-x}}{x} d x \int_{0}^{x}[y \varphi(y)-1] d y \\
=\int_{1}^{\infty} \frac{e^{-x}}{x} d x \int_{0}^{1}[y \varphi(y)-1] d y+O(\alpha) \\
=-2 \alpha \epsilon_{1}(1)\left(m+\frac{1}{2}\right) ! / m !+O(\alpha) . \\
\text { 5. } \int_{0}^{1} e^{-x} / \varphi(x)-1 / x / \chi(x) d x
\end{gathered}
$$

Expanding $\chi$ in power series and integrating, we get

$$
\begin{aligned}
\int_{0}^{1} & e^{-x}\left(\varphi(x)-\frac{1}{x}\right) \chi(x) d x=\int_{0}^{1}[x \varphi(x)-1] d x+O(\alpha) \\
& =-2 \alpha \frac{\left(m+\frac{1}{2}\right) !}{m !} .
\end{aligned}
$$

6. $\int_{0}^{1} e^{-x} / \varphi(x)-1 / x / \eta(x) d x$

$$
\begin{aligned}
& \int_{0}^{1} e^{-x}\left(\varphi(x)-\frac{1}{x}\right) \eta(x) d x \\
& \quad=\int_{0}^{1}\left(\varphi(x)-\frac{1}{x}\right) d x \int_{0}^{x}[y \varphi(y)-1] d y+O(\alpha), \\
& \alpha \int_{0}^{1 / \alpha}\left(W-\frac{1}{x}\right) d x \int_{0}^{x}[y W(y)-1] d y+O(\alpha) \\
& \quad=\alpha \int_{0}^{\infty}\left(W(x)-\frac{1}{x}\right) d x \int_{0}^{x}[y W(y)-1] d y+O(\alpha) .
\end{aligned}
$$

\section{APPENDIX C}

\section{Lower bound for $W_{m}$}

$(1 / m !) e^{-t} t^{m} d t$ is a probability measure and $\langle t\rangle$ $=m+1$. For fixed $x,\left(t+x^{2}\right)^{-1 / 2}$ is concave. By Jensen inequality

$$
\left\langle\left(t+x^{2}\right)^{-1 / 2}\right\rangle \geqslant\left(\langle t\rangle+x^{2}\right)^{-1 / 2}
$$

or

$$
W_{m}(x) \geqslant\left(m+1+x^{2}\right)^{-1 / 2} .
$$

Since $\left(a^{2}+b^{2}\right)^{1 / 2} \leqslant a+b$,

$$
W_{m}(x) \geqslant\left[(m+1)^{1 / 2}+x^{2}\right]^{-1} \text {. }
$$

$$
\text { 2. } 0 \leqslant f_{m} \leqslant 2(m+1)^{1 / 2}(1-\ln 2)
$$

$f_{m}$ is evidently positive [see (3.21) and (3.3)]. By (C1)

$$
\begin{aligned}
f_{m} & \leqslant \int_{0}^{\infty}\left(\left(m+1+x^{2}\right)^{-1 / 2}-\frac{1}{x}\right) d x \int_{0}^{x} d y\left[y\left(m+1+y^{2}\right)^{-1 / 2}-1\right] \\
& =(m+1)^{1 / 2} \int_{0}^{\infty}\left(\left(1+x^{2}\right)^{-1 / 2}-\frac{1}{x}\right)\left[\left(1+x^{2}\right)^{1 / 2}-x-1\right] d x \\
& =2(m+1)^{1 / 2}(1-\ln 2) .
\end{aligned}
$$

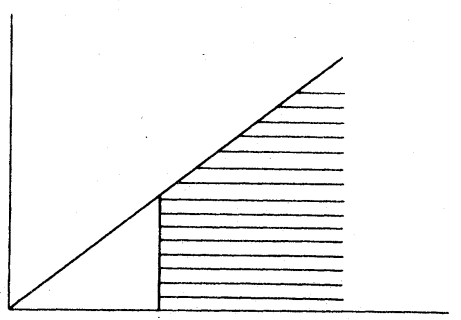

FIG. 1. Domain of integration for the double integral in Eq. (B8).

\section{APPENDIX D}

1. Upper and lower bounds for $\operatorname{Tr}(P)$

A lower bound is

$$
\begin{aligned}
\operatorname{Tr}(P) & \geqslant \int_{0}^{\infty} e^{-2 \alpha x}\left[(m+1)^{1 / 2}+x\right]^{-1} \\
& =e^{2 \alpha(m+1)^{1 / 2}} \epsilon_{1}\left[2 \alpha(m+1)^{1 / 2}\right] \\
& \geqslant \epsilon_{1}\left[2 \alpha(m+1)^{1 / 2}\right],
\end{aligned}
$$

where $\epsilon_{1}(x)$ is the exponential integral. For the upper bound write 


$$
\begin{aligned}
\operatorname{Tr}(P) & =\int_{0}^{1} W(x) e^{-2 \alpha x} d x+\int_{1}^{\infty} \frac{e^{-2 \alpha x}}{x} d x+\int_{0}^{\infty} e^{-2 \alpha x}\left(W(x)-\frac{1}{x}\right) d x \\
& =\int_{0}^{1} W(x) d x+\int_{1}^{\infty}\left(W(x)-\frac{1}{x}\right) d x+\epsilon_{1}(2 \alpha)+\int_{0}^{1}\left(e^{-2 \alpha x}-1\right) W(x) d x+\int_{1}^{\infty}\left(1-e^{-2 \alpha x}\right)\left(\frac{1}{x}-W(x)\right) d x \\
& \leqslant \ln 2+\epsilon_{1}(2 \alpha)+\ln \left[1+(m+1)^{1 / 2}\right]
\end{aligned}
$$

where we have used the negativity of the fourth term, (3.6) and (3.7), and finally (C3) to bound the last integral.

\section{Upper bound for $\operatorname{Tr}(P Q)$}

From (3.3) and (3.11)

$$
\operatorname{Tr}(P Q) \leqslant \int_{0}^{\infty} \frac{e^{-2 \alpha x}}{x} d x \int_{0}^{x} \frac{d y}{y}\left(1-e^{-2 \alpha y}\right)=\frac{\pi^{2}}{12}
$$

by (B1).

\section{APPENDIX E}

Truncated Coulomb

The binding $\alpha^{2}$ of the ground state of

$$
-\frac{d^{2}}{d x^{2}}-\frac{\lambda}{1+|x|}
$$

is given by

$$
\left.\frac{d W_{\beta}(x)}{d x}\right|_{x=2 \alpha}=0, \quad \beta=\frac{\lambda}{2 \alpha},
$$

where $\mathrm{re}^{15,16}$

$$
\begin{aligned}
W_{B}(x)=e^{-x / 2} & -\frac{1}{\beta}+\sum_{n=1}^{\infty} \frac{(1-\beta)_{n}}{n !(n+1) !} A_{n} x^{n+1} \\
& +[\ln x+\psi(1-\beta)-\psi(1)-\psi(2)] \\
& \left.\times \sum_{n=0}^{\infty} \frac{(1-\beta)_{n}}{n !(n+1) !} x^{n+1}\right),
\end{aligned}
$$

where

$$
(1-\beta)_{n}=(1-\beta)(2-\beta) \cdots(n-\beta), \quad(1-\beta)_{0}=1
$$

$$
\left.A_{n}=\sum_{j=0}^{n-1}[j+1-\beta)^{-1}-(j+1)^{-1}-(j+2)^{-1}\right] .
$$

A weak coupling expansion gives

$$
\alpha=\lambda \operatorname{Tr}(P)+\left(\lambda^{2} / \alpha\right) \operatorname{Tr}(P Q)+\cdots,
$$

where

$$
\begin{aligned}
& \operatorname{Tr}(P)=e^{2 \alpha} \epsilon_{1}(2 \alpha), \\
& \operatorname{Tr}(P Q)=\frac{1}{12} \pi^{2}-\alpha \ln ^{2}(2 \alpha)+O(\alpha)
\end{aligned}
$$

In $\mathrm{T}$ able IV a comparison is being made between the exact (E2) and (E5)-(E6) for values of $\lambda$ corresponding [Eq. (2.3)] to $B=100,200,283,500$, and 1000.
*Part IV of our series (Refs. 2, 3, and 17) "Schrödinger Operators in Magnetic Fields,"

${ }^{1}$ R. H. Garstang, Rep. Prog. Phys. 40, 105 (1977).

${ }^{2}$ J. E. Avron, I. W. Herbst, and B. Simon (unpublished).

${ }^{3}$ B. Simon, Ann. Phys. (N.Y.) 97, 279 (1976).

${ }^{4}$ R. Blankenbecler, M. L. Goldberger, and B. Simon, Ann. Phys. (N.Y.) 108, 69 (1977); M. Klaus, ibid. 108, 288 (1977); (unpublished).

${ }^{5}$ L. Schiff and H. Snyder, Phys. Rev. 55, 59 (1939).

${ }^{6}$ M. Ruderman, Phys. Rev. Lett. 27, 1306 (1971).

"E. R. Smith, J. R. W. Henry, G. L. Surmelian, and R. F. O'Connell, Phys. Rev. D $\underline{6}, 3700$
(1972).

${ }^{8}$ A. R. P. Rau and L. Spruch, Astrophys. J. 207B, 671 (1976).

${ }^{9}$ A. Galindo and P. Pascual, Nuovo Cimento 34B, 155 (1976).

${ }^{10}$ D. Cabib, F. Fabri, and G. Firoio, Nuovo Cimento 10,185 (1972).

${ }^{11} \mathrm{~V}$. Canuto and D. C. Kelly, Astrophys. Space Sci. 17, 277 (1972).

${ }^{12}$ A. R. P. Rau, L. Spruch, and R. Mueller, Phys. Rev. A 11, 1865 (1975); 11, 789 (1975).

${ }^{13}$ G. L. Surmelian and R. F. O'Connell, Astrophys. J. 190, 741 (1974).

${ }^{14}$ J. E. Avron, I. W. Herbst, and B. Simon, Ann. Phys. 
(N.Y.) 114, 431 (1978).

${ }^{15}$ R. Loudon, Am. J. Phys. 27, 649 (1959).

${ }^{16}$ L. K. Haines and D. H. Roberts, Am. J. Phys. 37, 1145 (1969).

${ }^{17} \mathrm{~J}$. E. Avron, I. W. Herbst, and B. Simon, Duke Math. J. 45,847 (1978).

${ }^{18} \mathrm{I}$. W. Herbst, Commun. Math. Phys. 53,285 (1977).

${ }^{19} \mathrm{R}$. Cohen, J. Lodenquai, and M. Ruderman, Phys. Rev. Lett. 25, 467 (1970).

${ }^{20}$ D. M. Larsen, J. Phys. Chem. Solids 29, 271 (1978).

${ }^{21}$ R. P. Mueller, A. R. P. Rau, and L. Spruch, Phys.
Rev. Lett. 26, 1136 (1971)

${ }^{22} \mathrm{G}$. Surmelian, $\mathrm{Ph} . \mathrm{D}$. thesis, Louisiana State University, 1973 (unpublished); D. Larsen (private communication).

${ }^{23} \mathrm{~B}$. Simon, Trace Ideals and Their Applications (Cambridge University, Cambridge, England, 1979).

${ }^{24}$ I. S. Gradshteyn and I. M. Ryznik, Table of Integrals Series and Products (Academic, New York, 1965); M. Abramowitz and I. A. Stegun, Handbook of Mathematical Functions (Dover, New York, 1964). 